\title{
On the PFQ-based Training Mode for the Special Force
}

\author{
Jianxin Yang \& Min Yu \\ Military Preventive Medicine Department \\ Fourth Military Medical University \\ Xi' an 710032, China
}

Fuyong Liu

Bethune International Peace Hospital

398 West Zhongshan Road, Shijiazhuang 050082, Hebei, China

Tel: 86-311-8799-8900 E-mail: liufy1065@hotmail.com

\begin{abstract}
The purpose of the research is to construct the theoretical framework of the physical fitness quotient, the assessment system, and the PFQ-based training mode. The methodology of the research includes the PFQ-related theories, assessments, literature review and consultancy. Result shows the feasibility of the construction of the PFQ-based assessment system, in particular, of the training system for the special force. It is concluded that the PFQ-based training mode is a trend for the special force.
\end{abstract}

Keywords: Physical fitness quotient, Physical fitness training, Special force

For the extensive use of the hi-tech weapons, the ways of future wars are expected to be substantially different. This requires the special force to adapt their physical fitness to the new military battles. Therefore, a reform should be performed on the physical fitness training in relation to the contents, forms and methods. In addition, it is argued that the scientific physical fitness training is vital for the special force. Theoretical and scientific research of the methodology is compulsory for the improvement of the physical fitness. Physical fitness quotient is a new assessment, for it helps to spot the proper gifts of individuals, and helpful to launch scientific trainings.

\section{An overview of the physical fitness trainings for the special force}

\subsection{Physical fitness}

Physical fitness refers to the physical strength, a force that an individual demonstrates in making a move. It is the fundamental and primary guarantee for the human labor, living and learning. The special force is a group of special individuals demanding special physical fitness from their unusual work. Likewise, the unusual physical strength that is compulsory for special force to adapt to the warfare should includes the physical movement, like running, jumping, and throwing, physical functions, like heart beats and lungs, and flexible capabilities to survive the complicated situations. The physical strength of the special force may be a significant component of the battling force, so to speak.

\subsection{The features of the physical fitness trainings for the special force}

It is true that the physical fitness status of an individual is greatly influenced by the hereditary factors, but it may surely be acquired by the later trainings. The approach is the especially prepared trainings aimed at the improvement of the physical fitness status of the army forces needed for the warfare. The trainings enable the army to maintain the strengthened battling force and ensure the proper performance of the warring skills. Special force forms a special community of the army, which demands an unusual status of physical fitness and warring versatility. Consequently, the courses of trainings are committed to unusual length, compact subjects and extreme tension, in addition to the purposeful missions.

\subsection{The modes of the physical fitness training for the special force}

The writers of the paper have reviewed the physical fitness status of the Chinese special force. The weaknesses may have impacts on the general battling force of the army. It is compulsory, therefore, the strengthening of the physical fitness and the trainings of the special force remain a realistic and urgent problem to resolve. 
The physical fitness training modes applied to the Chinese special force are basically imitated from those of the US army. The trainees receive, without exception, the trainings on vigor, rapidity, agility, endurance, flexibility, balancing or steadiness, dizziness resistance, and the like to adapt to the military fighting. Concrete measures are the 5-km long race, horizontal and parallel bars (1-2), 400-meter hurdle race, grenade throwing, push-up, and barbell pushing.

All this is helpful to the trainees with the general improvement of the physical fitness, but may not take advantage of the good points of the individuals, failing to match the practical drills or emergencies. So the mode needs reforming (Ke, J. and Lin, J. 2001.)( Ren, W. and Zhang, Y. 2000.)( Lei, E. and MAO Y. 2004.).

\section{The concept and classification of the physical fitness quotient}

\subsection{Concept}

The physical fitness quotient is a standardized system to assess the physical fitness to indicate the trends of health and the status of the basic physical fitness. The higher the physical fitness quotient is, the more safe and sound the health and the physical functions are. Therefore, the PFQ-based military training of the physical fitness has become a popular tendency.

\subsection{Classifications of physical fitness}

Generally speaking, the physical fitness of adults may be divided into three types, the health-related physical fitness, sport-related physical fitness, and skill-related physical fitness. Likewise, the physical fitness quotient may be divided into health-related, sport-related, and skill-related physical fitness quotient.

\subsubsection{The health-related physical fitness quotient}

The health-related physical fitness quotient refers to the physical quotient or competence of an individual to deal with the routine of work, enjoy the leisure and recreations, and handle the potential emergencies. The detailed components are the strength of muscle, endurance of muscle, pliability, resistance of heart and lungs, and the percentage of fat.

\subsubsection{The sport-related physical fitness quotient}

The sport-related physical fitness quotient is closely related to the basic sport or physical competence, but includes agility, stability, balancing, speed, and response, in addition to the components for the health-related physical fitness.

\subsubsection{The skill-related physical fitness quotient}

The skill-related physical fitness quotient refers to the special skill-related physical fitness that is imbedded into the sport skills to a certain extent. For instance, a basket-ball player demands such sport skills as the capabilities of speedy dribbling and passing, accurate and swift shooting. Due to the immediate affects of the types of sports, the physical fitness quotient of an individual is usually justified by the combined assessments of health-related and sport-related physical fitness quotients.

As far as the physical fitness trainings for the special force are concerned, attempts should be made to spot, by the assessment of the sport-related physical fitness quotient during the training, the individual physical quotient that is the physical ability to learn the sports. In addition, a developmental strategy is applied to suitable sports in accordance with the specific sport gifts to develop the physical fitness trend of the individual(Ke, J. and Lin, J. 2001.)( Ren, W. and Zhang, Y. 2000.)( Lei, E. and MAO Y. 2004.).

\section{The theoretical framework of PFQ-based physical fitness training mode for special force}

\subsection{Theoretical framework}

The writers of the paper argue for a hypothetic structure of PFQ (Figure 1) as the theoretical framework to meet the need for the PFQ-based physical fitness training mode for the special force in which the PFQ (physical fitness quotient) is HPFQ (health-related physical fitness quotient) and SPFQ (sport-related physical fitness quotient).

Given a high rate of HPFQ of an individual, the trend of the health is observed. By the same token, the high rate of SPFQ stands for sound competence of the sports. With a high rate of both HPFQ and SPFQ, an individual possesses brilliant physical health and sport physical fitness, and is thus apt to sport achievements and sport gifts. Moreover, the individual is expected to possess special potentials for a specific sport, should his specified sport components achieve a high assessment ratio. This special training for the special force is quite different from the traditional training designed for all.

\subsection{Methods of assessment}

On the basis of the theoretical framework, methods of assessment are constructed of the PFQ-based training for the special force with regard to the health-related and sport-related physical fitness quotients. The assessment is carried out in two different groups. In the HPFQ group, the assessment centers on the physical buildup, pliability, muscle strength, explosive force, and functions of heart and lungs, while in the SPFQ group, the focus of the assessment includes response to rod drop, and single-leg standing with eyes closed, in order to assess the special force in relation to the 
response, balancing, agility, speed, and stability (Figure 2)( Shi Y. 2002.)( Liu J. and ZHANG, J. 2002.)

\section{Mode of the PFQ-based training for the special force}

On the basis of the PFQ-based theory and assessment methods, the mode of the PFQ-based training is hypothesized for the special force. The flowing chart starts with the establishment of the assessment system of the physical fitness quotient, assessment in operation, designation of the specific sports for individual of the special force, and PFQ-based training in operation. Should there be problems in the course of training, they are fed back in time, so the assessment system is continually improved toward the set up of the PFQ-based training system of the special force (Figure 3)(Lin J. 2004.).

In conclusion, the PFQ-based training system of the special force is expected to greatly enhance the quality of the newly-recruited, which is helpful to the progress of the systematization, standardization, and scientific normalization. Furthermore, a timely understanding and objective assessment is achieved of the physical fitness and battling force, in particular, of the special force. The theory may serve as a guide for the PFQ-based training. Therefore, it is proposed that the PFQ-based monitoring and feedback mechanisms, centers or networks be established throughout the military zones, so that the physical tests may be regularly performed and reported to the headquarters of the military commission. The ultimate purpose of the paper is to enable the physical fitness training to be scientific and effective to provide with the theoretical and scientific basis for the physical training and improvement of the physical strength of the military force.

\section{References}

Ke, J. and Lin, J. (2001). A developmental survey of the physical fitness of the citizens and soldiers. PLA College of Physical Education, 20, 6-9.

Lei, E. and MAO Y. (2004). Perspectives of the comprehensive protection of the military health, Military Medical University,28,169-170.

Lin J. (2004). Construction of the Chinese military physical fitness, PLA College of Physical Education, 23,1-5.

Liu J. and ZHANG, J. (2002). A tentative study of the comprehensive assessment of physical fitness, PLA College of Physical Education, 21,87-90.

Ren, W. and Zhang, Y. (2000). Reflections on the physical fitness training in the military force. PLA College of Physical Education, 19,53-56.

Shi Y. (2002). On the new methods of assessment of the physical fitness, First Military Medical University, Postgraduate Papers.

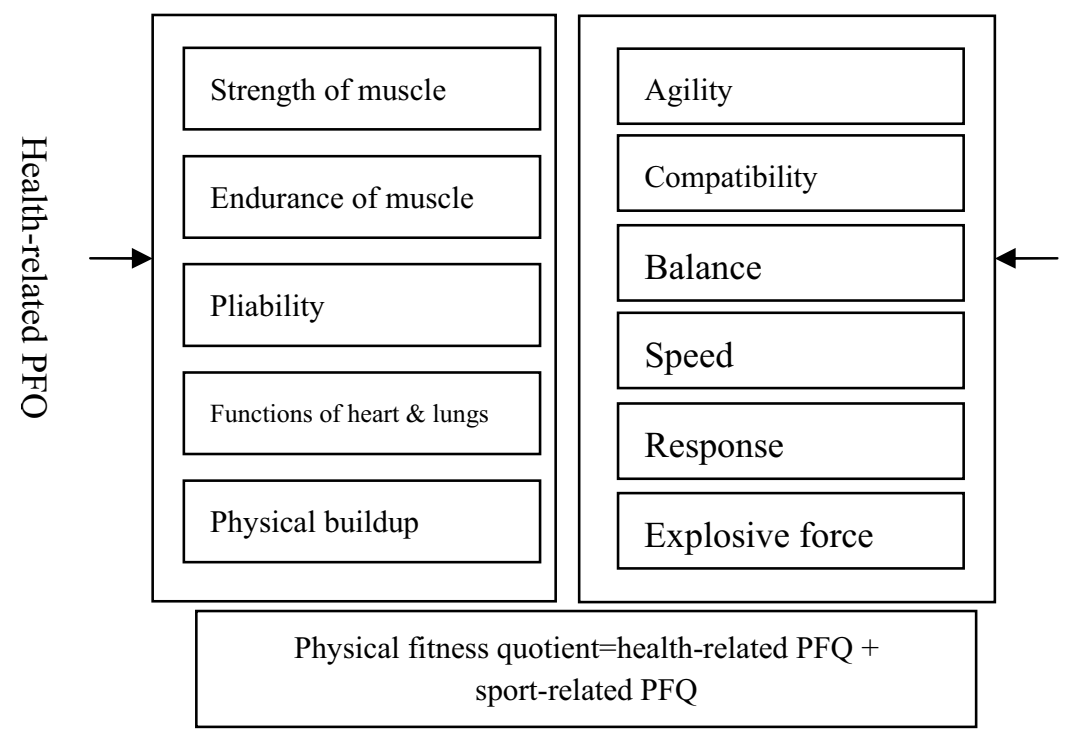

Figure 1. Diagram of the Structure of PFQ 


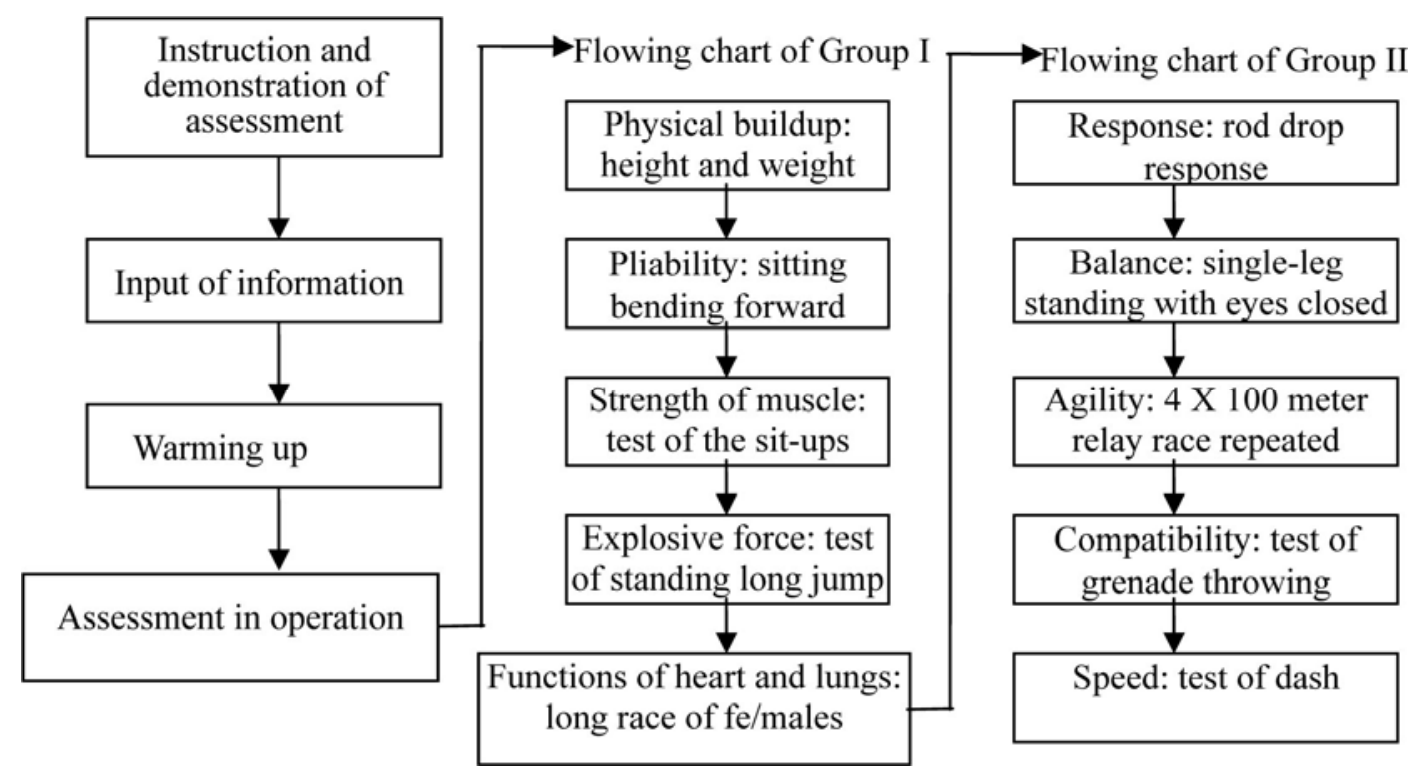

Figure 2. Methods of Assessment of the PFQ-based training for the special force

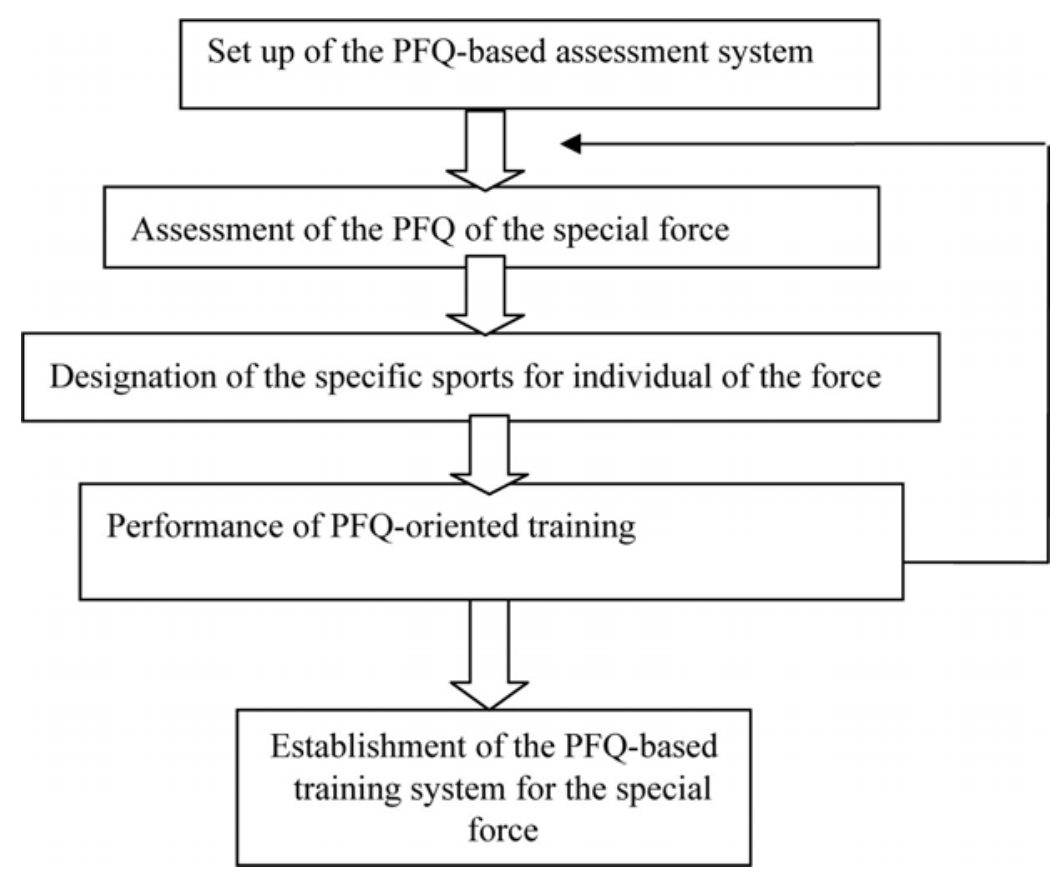

Figure 3. PFQ-based training mode for the special force 